\title{
Predictive Modeling of Cardiac Fiber Orientation Using the Knutsson Mapping
}

\author{
Karim Lekadir, Babak Ghafaryasl, Emma Muñoz-Moreno, Constantine Butakoff, \\ Corné Hoogendoorn, and Alejandro F. Frangi \\ Center for Computational Imaging \& Simulation Technologies in Biomedicine \\ Universitat Pompeu Fabra and CIBER-BBN, Barcelona, Spain \\ karim.lekadir@upf.edu
}

\begin{abstract}
The construction of realistic subject-specific models of the myocardial fiber architecture is relevant to the understanding and simulation of the electromechanical behavior of the heart. This paper presents a statistical approach for the prediction of fiber orientation from myocardial morphology based on the Knutsson mapping. In this space, the orientation of each fiber is represented in a continuous and distance preserving manner, thus allowing for consistent statistical analysis of the data. Furthermore, the directions in the shape space which correlate most with the myocardial fiber orientations are extracted and used for subsequent prediction. With this approach and unlike existing models, all shape information is taken into account in the analysis and the obtained latent variables are statistically optimal to predict fiber orientation in new datasets. The proposed technique is validated based on a sample of canine Diffusion Tensor Imaging (DTI) datasets and the results demonstrate marked improvement in cardiac fiber orientation modeling and prediction.
\end{abstract}

\section{Introduction}

The study of fiber structure in the myocardium is of considerable importance for the understanding of its electro-mechanical behavior and associated cardiac pathologies. Over the years, there has been an increasing interest in the use of Diffusion Tensor Imaging (DTI) for the measurement of cardiac fiber orientation [1]. However, the modality is currently infeasible in vivo due to its great sensitivity to cardiac motion. On the other hand, myocardial fibers tend to be arranged according to consistent patterns across individuals yet they are crucial in determining electrical propagation and contraction patterns. As a result, computational techniques are required to construct accurate subject-specific models of myocardial fiber orientation from ex vivo datasets so as to enable realistic simulation of the electro-mechanical function of the heart. Existing research emphasizes the need for modeling techniques that can encode the relationship between myocardial shape and fiber orientation, as well as that can take into account the inter-subject variability in fiber structure [1]. Amongst existing methods, a synthetic fiber model has been used in [2], which provides only the overall trend of the structure and thus lacks accuracy in subject-specific modeling. The work in [3] estimates fiber structure by warping the data to a predefined single template but the method is not based on a sample population and thus does not take 
into account the variability found in fiber orientation and shape. The Streeter model [4], on the other hand, is built from a database of histological datasets and thus far remains the most established model in cardiac simulation. The technique fits to the training data parametric equations relating the position in the myocardium with a selected set of global and local shape information, such as the left ventricular axis and position relative to the myocardial walls. It can be argued, however, that a significant amount of shape information is ignored, thus producing a model that might not approximate well enough the complex distribution of the fibers.

This paper presents an alternative approach through which the relationship between myocardial morphology and fiber orientation is statistically encoded based on a training sample population. To this end, the proposed technique firstly introduces a representation of the fibers in a 5D space that is continuous and distance preserving, thus allowing consistent manipulation of the orientation data. Additionally, the technique makes use of all the available shape information and extracts the latent directions in the shape space that correlate most with the fiber orientation. A nonlinear regression technique is then used to accurately estimate fiber orientation in new datasets based on the myocardial morphology. The proposed predictive model is validated based on a sample of normal canine cardiac DTI datasets.

\section{Methods}

\subsection{Dataset Description and Preprocessing}

To construct a predictive model of the myocardial fiber orientations with the proposed approach, we use a database of seven normal ex vivo DTI datasets of canine hearts (Center of Cardiovascular Bioinformatics and Modeling, at John Hopkins University). The images were produced using a 1.5T GE CV/I MRI scanner with an enhanced gradient system, $40 \mathrm{mT} / \mathrm{m}$ maximum gradient amplitude and a $150 \mathrm{~T} / \mathrm{m} / \mathrm{s}$ slew rate. The first eigenvector of the obtained DTI tensors was calculated to estimate the 3D vector describing the fiber orientation at each voxel. Furthermore, the predictive model proposed in this paper is aimed at encoding the statistical relationship between myocardial shape and fiber orientation. As a result, it is required to build surface meshes of the myocardial shapes and volumetric meshes of the myocardial fibers that correspond between the training datasets. To this end, the left ventricular walls are firstly segmented in each of the DTI volumes over the maps of Fractional Anisotropy (FA) using a semi-automatic tool [5]. The point correspondence between the obtained surface meshes is established based on the position of key anatomical features (i.e., mitral valve, apex, and aorta). The mean shape is subsequently computed and used to derive a volumetric mesh that contains the fiber orientation at uniformly distributed nodes within the myocardium. Finally, to obtain the fiber information at corresponding locations of the myocardium, the mean volumetric mesh is then mapped onto all image volumes by using the Thin Plate Spline (TPS) technique [6].

\subsection{Knutsson Mapping of Fiber Orientation}

To enable a consistent manipulation of the data within the proposed predictive model, it is important to use a suitable representation of the fiber orientations. The $3 \mathrm{D}$ vectors 
obtained from the diffusion tensors are non-directional and thus opposite vectors can represent the same fiber orientation. This introduces a fundamental ambiguity that must be specifically handled to avoid potential errors in the statistical analysis. In $2 \mathrm{D}$, this is resolved easily by using the double angle trick, i.e., the new orientation coordinates are the cosine and sinus of twice the angle between the line and a fixed coordinate axis. In 3D, however, the situation is more complicated and the solution presented in [7] involves a mapping to a 5D space with interesting mathematical properties. Given a unit vector characterized by the angles $\theta, \phi$ of its spherical representation, the obtained Knutsson function $M_{K}: R^{3} \rightarrow R^{5}$ can be derived as [7]:

$$
\begin{aligned}
& s=\sin ^{2} \theta \cos 2 \phi \\
& t=\sin ^{2} \theta \sin 2 \phi \\
& u=\sin 2 \theta \cos \phi \\
& v=\sin 2 \theta \sin \phi \\
& w=\sqrt{3}\left(\cos ^{2} \theta-\frac{1}{3}\right)
\end{aligned}
$$

The Knutsson coordinates $s, t, u, v, w$ essentially map half of a sphere onto a 5D space such that the surface is uniformly stretched in all directions and all pairs of opposite vectors are mapped onto the same location in the 5D space. The mapping described in Eq. (1) is therefore unique and removes the ambiguity found in directional vectors. More importantly, it can be shown that the Knutsson mapping induces a continuous representation that preserves distance [8] (thus the importance of the normalizing constants in the definition of the coordinate $w$ in Eq. (1)). Applications of the Knutsson mapping in the literature include curvature estimation in 3D images [8] and the analysis of high angular diffusion tensors of the brain [9].

With this representation, operations on the cardiac fiber orientations can be carried out consistently and the results need to be mapped back to the original 3D space, for example for visualization and simulation purposes. This can be carried out by first approximating the product $\mathbf{x x}^{T}$ from the mapping $M_{K}$ as follows:

$$
\mathbf{x x}^{T}=\frac{1}{2}\left(\begin{array}{ccc}
\frac{2-\sqrt{3} w}{3}+s & t & u \\
t & \frac{2-\sqrt{3} w}{3}-s & v \\
u & v & \frac{1+\sqrt{3} w}{3}
\end{array}\right)
$$

Subsequently, it can be then demonstrated that the 3D orientation can be derived from the principal eigenvector of $\mathbf{x x}^{T}$ [10]. 


\subsection{Nonlinear Predictive Modeling}

Existing research on cardiac fiber architecture suggests that a strong relationship exists between myocardial morphology and fiber structure. It is not trivial, however, to define for each region of the myocardium the shape information that is most suitable to estimate the corresponding fiber orientations. In the Streeter model [4], for example, it is assumed that the fibers are better described with respect to a number of preselected variables, such as the left ventricular axis and relative position to the myocardial walls. As a result, a substantial amount of available shape information is simply ignored with such approach, which can potentially limit the accuracy of the model. In this paper, the aim is to use all available shape information and automatically extract the variables in the shape space that are most relevant to the estimation of fiber orientation based on statistical criteria. In other words, the axes of shape variation that correlate the most with the fiber orientation maps need to be extracted and used to optimize the prediction capability of the obtained model.

Partial Least Squares (PLS) regression [11] is a dimensionality reduction technique with several advantages that make it ideal for the predictive modeling task of this paper. It can produce efficient regression models in situations where the number of predictors is very large and at the same time the training sample is small (thus introducing singular data matrices). This is beneficial for predictive modeling of fiber orientation due to the generally limited availability of ex vivo DTI datasets. In the following, let $\mathbf{X}$ be the data matrix representing the aligned myocardial shapes in the training set and $\mathbf{Y}$ the matrix of the 5D Knutsson data representing the fiber structures inside the myocardium. The aim of partial least squares regression is to perform a simultaneous decomposition of $\mathbf{X}$ and $\mathbf{Y}$ such that the score vectors obtained along the new representation axes of both the input and output matrices correlate most. One solution to the problem can be obtained through the NIPALS algorithm [11], which iteratively estimates new vectors $\mathbf{a}$ and $\mathbf{b}$ as:

$$
[\operatorname{cov}(\mathbf{X a}, \mathbf{Y b})]^{2}=\max _{\|\boldsymbol{c}\|=\|\mathbf{d}\|=1}[\operatorname{cov}(\mathbf{X c}, \mathbf{Y d})]^{2}
$$

At each iteration, the data matrices $\mathbf{X}$ and $\mathbf{Y}$ are deflated by subtracting the contribution along the latent axes found at previous step. At convergence, the predictors and outputs are decomposed as $\mathbf{X}=\mathbf{T} \mathbf{P}^{T}+\mathbf{E}$ and $\mathbf{Y}=\mathbf{U} \mathbf{Q}^{T}+\mathbf{F}$, respectively, where $\mathbf{P}$ and $\mathbf{Q}$ are the extracted latent vectors. The final step is to estimate the regression coefficient matrix $\mathbf{D}$ and the predictive model is obtained as:

$$
\hat{\mathbf{Y}}=\mathbf{T D Q} \mathbf{Q}^{T}
$$

It is important to note that only a subset of the latent vectors are used in the prediction and these are selected using cross validation such that they minimize the reconstruction of the output data in the training.

The mapping introduced in this paper for the representation of fiber orientation, as well as the nature of the data, can introduce a nonlinear interdependency between the matrices $\mathbf{X}$ and $\mathbf{Y}$. As a result, it is more appropriate to use a nonlinear implementation of the partial least square algorithm. Using the kernel version developed in [12], this can be achieved with a kernel transformation of the input data 
followed by the application of the linear PLS technique described above. The kernel function $\Phi$ used for this purpose is typically defined as Gaussian or polynomial. The kernel gram matrix $\mathbf{K}=\Phi \Phi^{T}$ of the cross product between all input data points is computed and used to obtain the final nonlinear predictive model as follows:

$$
\hat{\mathbf{Y}}=\mathbf{K}_{t} \mathbf{U}\left(\mathbf{T}^{T} \mathbf{K U}\right)^{-1} \mathbf{T}^{T} \mathbf{Y}
$$

where $\mathbf{K}_{t}$ is the kernel Gram test matrix estimated from the new input data. More details regarding the kernel PLS implementation can be found in [11].

\section{Results}

The proposed predictive model is validated using the seven DTI datasets of normal canine hearts described in Section 2.1 on a leave-one-out basis. The absolute value of the dot product between the predicted fiber orientations and the original data is calculated to measure the extent of agreement and the accuracy of the proposed technique. A value close to 0 indicates strong disagreement, while a dot product close to 1 indicates high prediction accuracy. Firstly, we applied a template warping approach between all pairs of datasets similarly to the work in [3] and using TPS warping. We found the results to be inconsistent depending on the choice of the subject to serve as a template, with a similarity measure equal to 0.47 for subject 5 versus subject 1 and equal to 0.85 for subject 4 versus subject 1 . This justifies well the use of a statistical approach as presented in this paper, where the variability in fiber orientation between the datasets is specifically modeled.

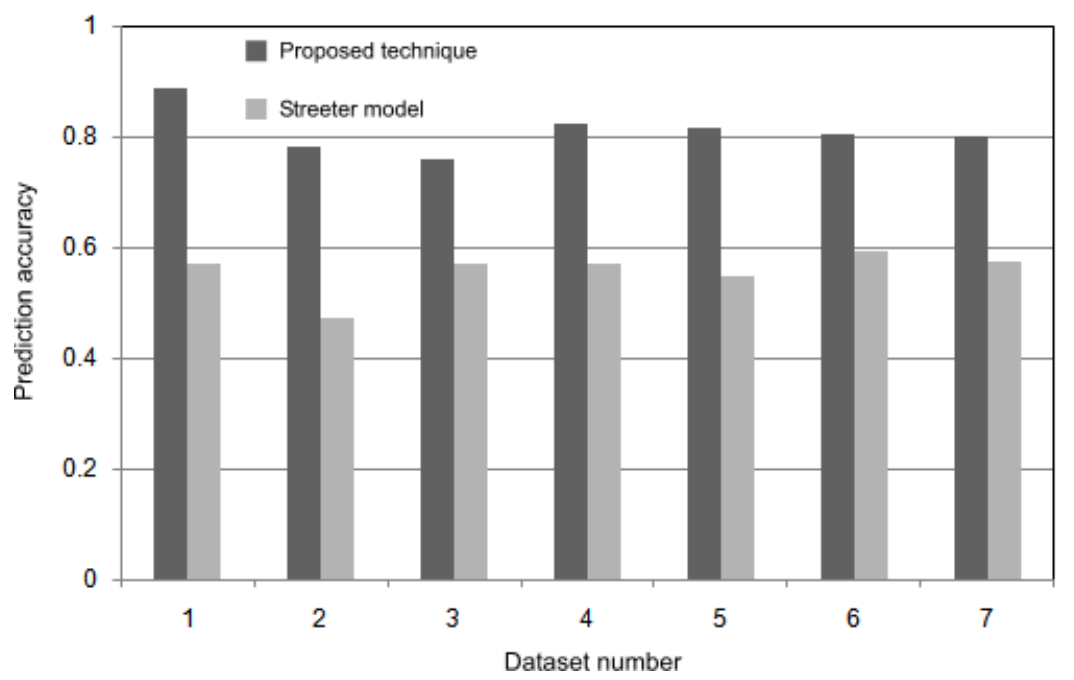

Fig. 1. Dot product accuracy results for the seven canine DTI images using the Streeter model and the proposed technique, showing significant improvement throughout all datasets 


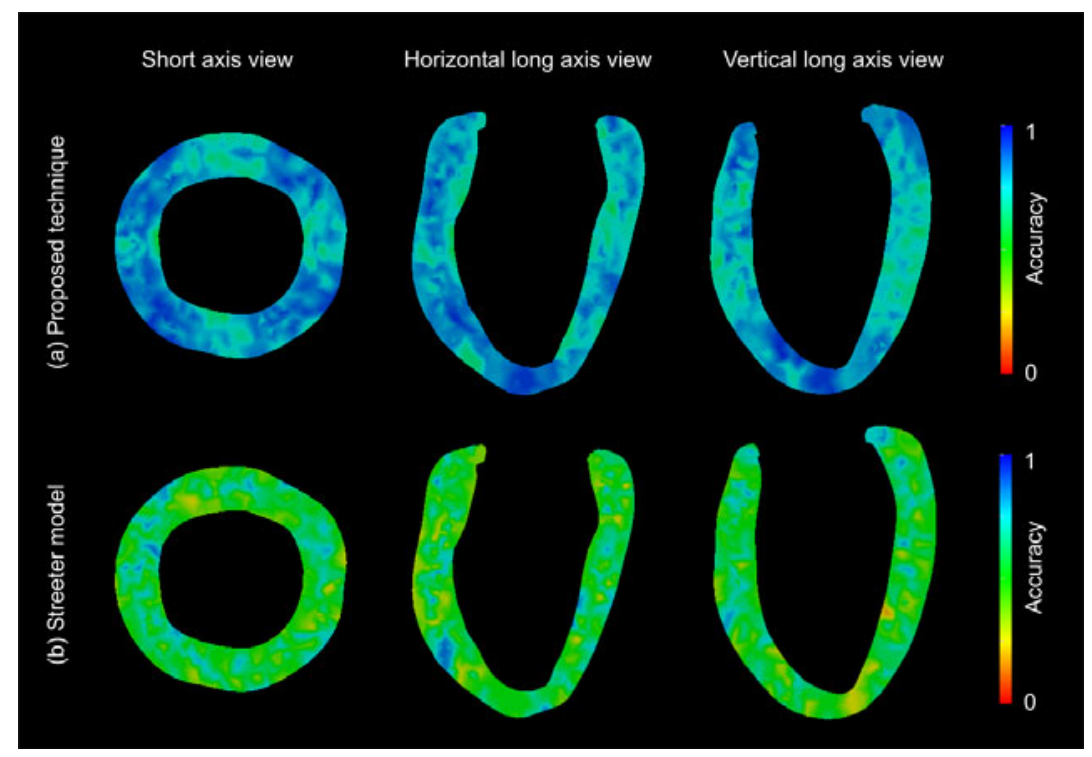

Fig. 2. Average accuracy maps for the proposed technique and the existing Streeter model

Furthermore, the Streeter model [4] was implemented and tested against the accuracy obtained with the approach presented in this paper. The results for both techniques are plotted in Fig. 1, where it can be seen that the proposed method improves the prediction of fiber orientation for all datasets. These results are significant particularly given the fact that the predictive models were built from a small training set, which shows the performance capability of the non-linear PLS method in this work. Table I summarizes the average, standard deviation and minimal accuracy results, where it can be observed that the proposed predictive model is not only accurate but also consistent throughout all datasets, with an average accuracy equal to 0.81 and a minimal accuracy of 0.75 . It is also worth noting from Table I how the introduction of the Knutsson mapping for the representation of fiber orientation (third column) and the use of the nonlinear kernel technique for prediction (fourth column of the table) increase the accuracy of the model.

Table 1. Prediction accuracy using the proposed technique

\begin{tabular}{c|c|c|c|c}
\hline Accuracy & $\begin{array}{c}\text { Streeter } \\
\text { model }\end{array}$ & $\begin{array}{c}\text { L-PLSR in } \\
\text { 3D space }\end{array}$ & $\begin{array}{c}\text { L-PLSR in } \\
\text { Knutsson space }\end{array}$ & $\begin{array}{c}\text { NL-PLSR in } \\
\text { Knutsson space }\end{array}$ \\
\hline Average & 0.55 & 0.71 & 0.75 & 0.81 \\
Std & 0.06 & 0.08 & 0.06 & 0.04 \\
Minimum & 0.47 & 0.65 & 0.68 & 0.75 \\
\hline
\end{tabular}


For detailed visualization of the performance of the proposed technique, the average prediction accuracy for each fiber location in the myocardium is displayed in Fig. 2 for different long- and short-axis views. It can be seen that the fiber orientation prediction is accurate consistently for all regions of the myocardium, unlike the Streeter model which displays less accurate results. This is also due to the fact that the Streeter technique uses the same parametric model for all regions of the myocardium. On the contrary, the proposed technique extracts the latent structures most relevant for the prediction of each individual fiber orientation, which is a natural approach since the relationship between shape and fiber is expected to vary for different regions of the myocardium. Finally, an illustration of the predicted fiber orientations using the proposed technique is given in Fig. 3, where significant agreement with the original DTI data can be observed in all views and regions.

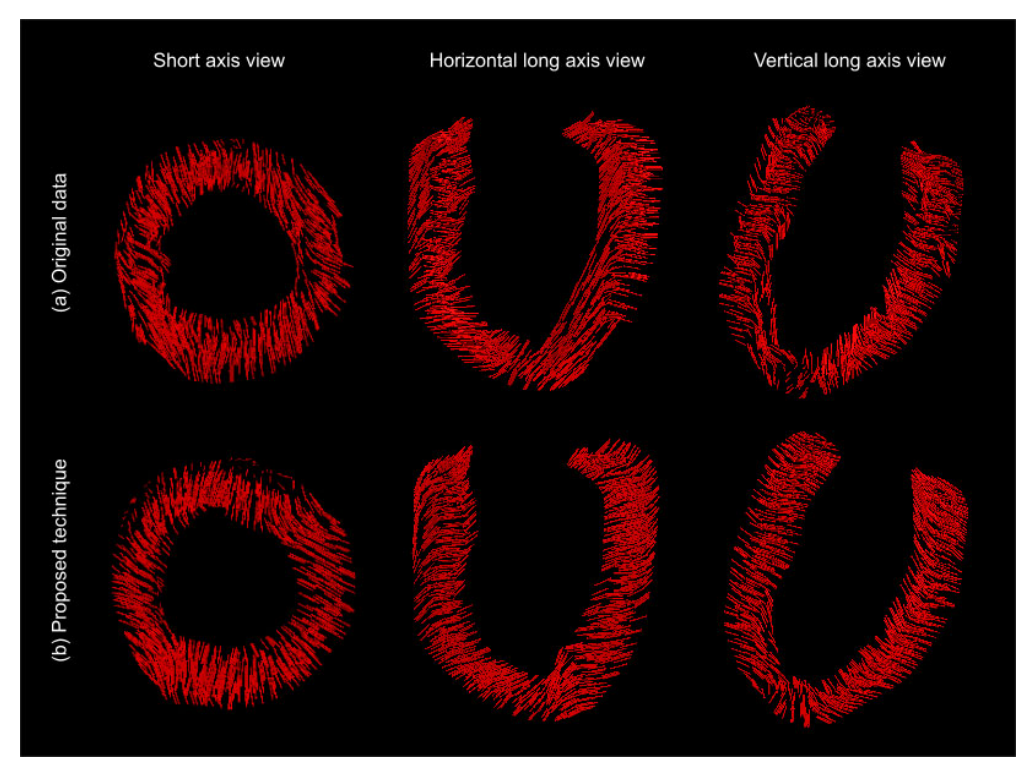

Fig. 3. Illustration of the fiber structure obtained by the proposed technique (b) as compared to the ground truth (a) derived from the original DTI data

\section{Conclusions}

Our conclusions following the work presented in this paper are twofold: firstly, the relationship between myocardial shape and fiber orientation is significant yet it is not trivial. Therefore it must be defined automatically and statistically by extracting the optimal latent structures from a training sample population. Secondly, the representation of orientation data in general, and particularly for the study of fiber structure, requires continuous and distance preserving mappings in order to allow for consistent statistical analysis, such as by using the Knutsson mapping described in this work. The initial results reported in this paper show the potential of the technique for fiber structure modeling and prediction. 
Acknowledgement. This work was funded by the CENIT program of the CDTI, the Industrial and Technological Development Centre of Spain, under the research project cvREMOD (ref. CEN20091044).

\section{References}

1. Peyrat, J.-M., Sermesant, M., Pennec, X., Delingette, H., Xu, C., McVeigh, E.R., Ayache, N.: Towards a statistical atlas of cardiac fiber structure. In: Larsen, R., Nielsen, M., Sporring, J. (eds.) MICCAI 2006. LNCS, vol. 4190, pp. 297-304. Springer, Heidelberg (2006)

2. Sermesant, M., Rhode, K., Sanchez-Ortiz, G.I., Camara, O., Andriantsimiavona, R., Hegde, S., Rueckert, D., Lambiase, P., Bucknall, C., Rosenthal, E., Delingette, H., Hill, D.L.G., Ayache, N., Razavi, R.: Simulation of cardiac pathologies using an electromechanical biventricular model and XMR interventional imaging. Medical Image Analysis 9(5), 467-480 (2005)

3. Sundar, H., Shen, D., Biros, G., Litt, H., Davatzikos, C.: Estimating myocardial fiber orientation by template warping. In: IEEE International Symposium on Biomedical Imaging (2006)

4. Streeter, D.: The cardiovascular system: gross morphology and fiber geometry of the heart, in Handbook of physiology. Williams \& Wilkins, Baltimore (1979)

5. Larrabide, I., Omedas, P., Martelli, Y., Planes, X., Nieber, M., Moya, J.A., Butakoff, C., Sebastián, R., Camara, O., Craene, M.D., Bijnens, B., Frangi, A.F.: GIMIAS: an open source framework for efficient development of research tools and clinical prototypes. In: Functional Imaging and Modeling of the Heart (2009)

6. Bookstein, F.L.: Principal warps: thin-plate splines and the decomposition of deformations. IEEE Transactions on Pattern Analysis and Machine Intelligence 11(6), 567-585 (1989)

7. Knutsson, H.: Producing a continuous and distance preserving 5-D vector representation of 3-D orientation. In: IEEE Computer Society Workshop on Computer Architecture for Pattern Analysis and Image Database Management, Miami Beach, Florida (1985)

8. Rieger, B., Timmermans, F.J., Vliet, L.J.v., Verbeek, P.W.: Curvature estimation of surfaces in 3D grey-value images. In: International Conference on Pattern Recognition, ICPR (2002)

9. Bhalerao, A., Westin, C.-F.: Hyperspherical von Mises-Fisher Mixture (HvMF) Modelling of High Angular Resolution Diffusion MRI. In: Ayache, N., Ourselin, S., Maeder, A. (eds.) MICCAI 2007, Part I. LNCS, vol. 4791, pp. 236-243. Springer, Heidelberg (2007)

10. Knutsson, H.: Representing local structure using tensor. In: Scandinavian Conference in Image Analysis, Oulu, Finland (1989)

11. Abdi, H.: Partial least squares regression (PLS-regression), in Encyclopedia for research methods for the social sciences. Sage, Thousand Oaks (2003)

12. Rosipal, R., Trejo, L.J.: Kernel patial least squares regression in reproducing kernel Hilbert space. Journal of Machine Learning Research 2, 97-123 (2002) 